\title{
Meta-analysis of variant rs 7903146 of TCF 7L 2 gene with secondary complications of Type 2 Diabetes Mellitus
}

\author{
Kanhaiya Singh ${ }^{1}$, NK Agrawal ${ }^{2}$, SK Gupta ${ }^{3}$, Kiran Singh ${ }^{1} *$ \\ ${ }^{1}$ Department of Molecular \& Human Genetics, Banaras Hindu University, Varanasi-221005, India. \\ ${ }^{2}$ Department of Endocrinology and Metabolism, Institute of Medical Sciences, Banaras Hindu University, Varanasi-221005, India. \\ ${ }^{3}$ Department of Surgery, Institute of Medical Sciences, Banaras Hindu University, Varanasi-221005, India.
}

\section{ARTICLE INFO}

Article history:

Received on: 15/09/2014

Revised on: 24/09/2014

Accepted on: 14/10/2014

Available online: $30 / 10 / 2014$

\section{Key words:}

Transcription factor 7-like 2

(TCF7L2), Polymorphism,

T2DM, secondary

complications, Meta analysis

\begin{abstract}
Persistent hyperglycemia in patients with Type 2 diabetes mellitus is driving force for developing secondary complications like neuropathy, retinopathy, nephropathy, cardiomyopathy and wound healing impairment in them. Other genetic risk factor includes variants of transcription factor 7-like 2 (TCF7L2) gene, which have been shown to be associated with these several secondary complications individually. The present study represents a systematic review and Meta analysis using electronic databases like PubMed, OMIM, ISI web of science and Embase to see the role of rs7903146 C > T variant of the TCF7L2 gene with secondary complications of T2DM. Data was collected using literature-based searching to perform a Meta analysis to pool the odds ratio (OR). This Meta analysis comprised of a total of 2060 cases with secondary complication of T2DM and 3049 age matched controls. Publication bias and study-between heterogeneity were also evaluated. The present Meta analysis of included studies showed a highly significant association of risk genotype TT with development of secondary complications in T2DM.
\end{abstract}

\section{INTRODUCTION}

Type 2 diabetes Mellitus (T2DM) is a chronic non communicable disease which is proving to be a leading cause of morbidity and mortality, especially in low income developing countries. In India alone, the prevalence of T2DM has increased about two fold from 32 million in 2000 to 62 million in the last decade [1]. World Health Organization and International diabetes foundation claim T2DM as the most challenging problem of $21^{\mathrm{st}}$ century due to secondary complications like cardiovascular disease, neuropathy, nephropathy and retinopathy associated with it [2]. The etiology of T2DM is generally polygenic but recent researchers have identified certain variants like PPARG Pro12Ala and $K C N J 11$ E23K which lead to monogenic form of diabetes [3]. Apart from candidate gene approaches, some hypothesis free genome wide analyses are also being done in T2DM cases to screen the common variants present in the population. One such genome wide study revealed the possible involvement of APC/Ccatenin/TCF pathway, commonly called Wnt pathway, in the patho-physiology of T2DM [4]. Wnt is a gene related to developmental pathway and it play important roles in embryonic

\footnotetext{
* Corresponding Author

Kiran Singh, Department of Molecular \& Human Genetics Banaras Hindu University, Varanasi-221005, India

E-mail: skiran@bhu.ac.in, singhk4@ rediffmail.com
}

induction, cell polarity generation and in the specification of cell fate. They have also been shown to work in co-ordination with matrix metalloproteinases which are also involved in regulation of wound healing [5].

Recent reports suggest that Wnt signaling brings about the movement and differentiation of cardiac fibroblasts following myocardial infarction [6]. Downstream agents of wnt signaling like $\beta$-catenin and c myc help in the movement of epidermal stem cells to the wound site during wound healing [7]. The transcription factor 7 like 2 gene (TCF7L2) product is a High mobility group box transcription factor and it is a part of $\beta$-catenin signaling cascade [8]. This transcription factor is guided by $\beta$-catenin and helps in the transcription of downstream genes like PPAR- $\gamma$, Myc, Cyclin D1 etc. and probably affect $\beta$-cell function either by modulating $\beta$-cell response to glucose or by modulating the incretin action or secretion [9].

Multiple intronic single nucleotide polymorphisms (SNPs) in TCF7L2 gene have shown to be associated in several European, African, American and Asian cohorts of world including Indians, although a marked variation in allele frequencies is seen due to difference in ethnic descend. Re-sequencing and comparative association studies in search of putative element revealed that rs7903146 is a most likely casual variant SNP located in intron 4 of TCF7L2 gene. Being situated in intronic region this variant likely 


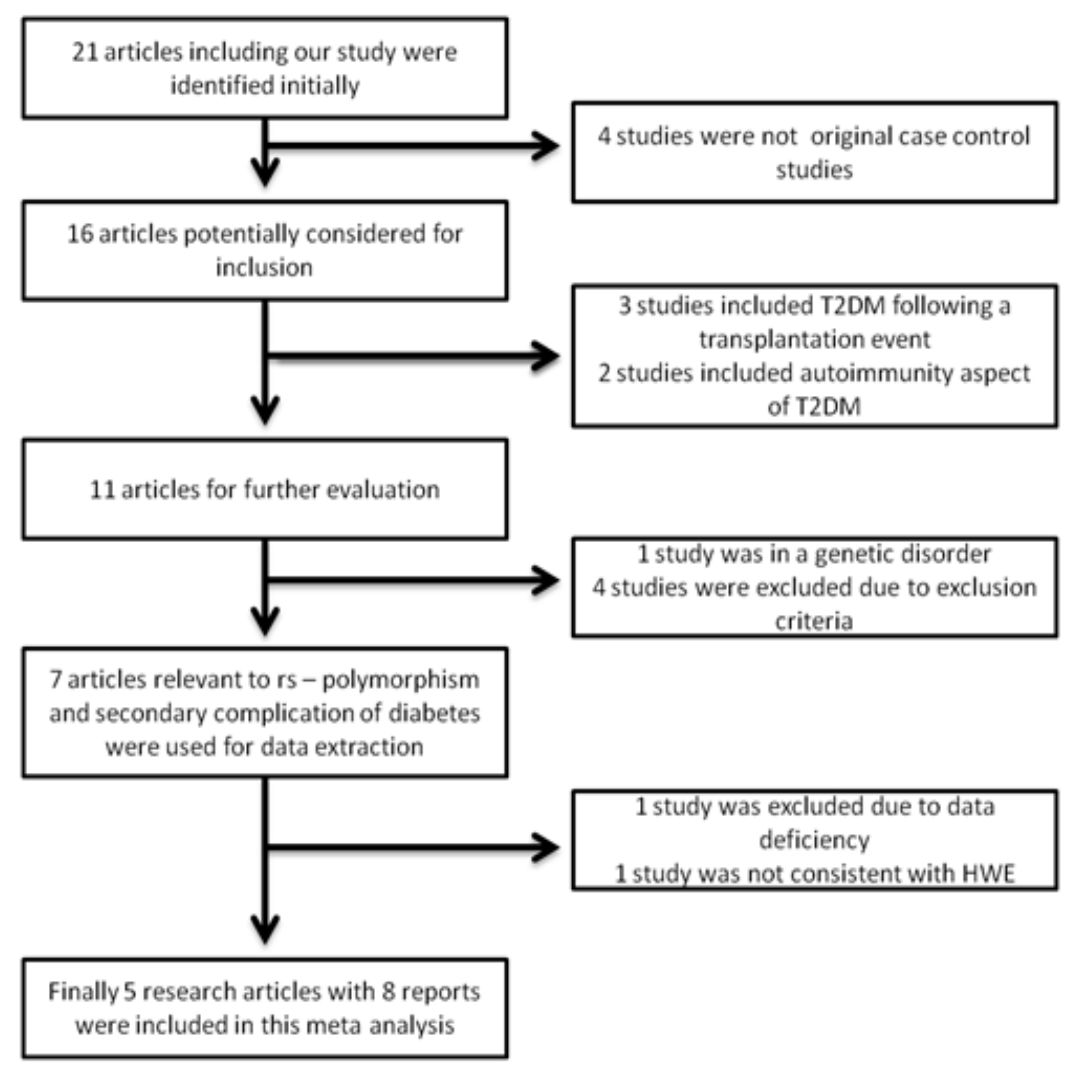

Fig. 1: Data sheet for Meta analysis- genotype data of studies in secondary complications of T2DM. Genotype data for SNP rs 7903146 polymorphism were extracted according to standard extraction form as shown in the figure. A total of 5 research articles comprised of 8 studies including our previous study were found to be eligible on our search criteria.

Mota Shorrame

\begin{tabular}{|c|c|c|c|c|c|}
\hline & $\begin{array}{l}\text { Odts } \\
\text { ratio }\end{array}$ & $\begin{array}{l}\text { Lover } \\
\text { limit }\end{array}$ & $\begin{array}{l}\text { Uper } \\
\text { limit }\end{array}$ & ZPalue & pNalue \\
\hline Buraczy nska Metal. 2011 & 3238 & 2.330 & 4.500 & 699 & \\
\hline Buraczynska Metal. 2011 a & 2758 & 1960 & 3.882 & 5.820 & \\
\hline Buraczynska Metal. 20110 & 2676 & 1964 & 3.648 & 6.231 & \\
\hline Cocaccl Cet al. 2012 & 3266 & 1.471 & 7.250 & 2909 & \\
\hline Cocacel Cet al. $2012 a$ & 3015 & 1.337 & 6.796 & $2 ळ 1$ & \\
\hline Mendeln A et al. 2011 & 1825 & 1.034 & 3222 & 2.075 & \\
\hline SousaA etal. 2009 & 1.264 & 0.812 & 1967 & 1.096 & \\
\hline \multirow[t]{3}{*}{ Kannaly a etal. 2013} & 2217 & 1.032 & 4.763 & 2041 & \\
\hline & 2499 & 2.139 & 2920 & 11.526 & \\
\hline & 2422 & 1909 & 3.074 & 7.276 & \\
\hline
\end{tabular}

\section{Statisticsfor mchqudy}

Oets rato ard $95 \% \mathrm{Cl}$

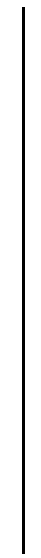

0.1

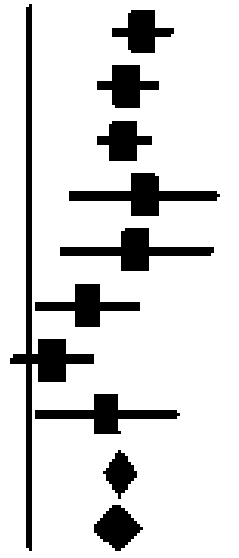

1
10

Fig. 2: Odds ratio of each study and the pooled estimate is shown by the forest plot. Cochran's $\chi^{2}$ test ( $Q$ test) was used to evaluate heterogeneity between studies; a threshold $\mathrm{P}$ value of 0.1 was considered statistically significant, and the inconsistency value $\left(\mathrm{I}^{2}\right)$ test was also conducted to evaluate heterogeneity of studies. The ' $I^{2}$ ' value of $49.70 \%$ for between studies comparison showed low level of true heterogeneity. The fixed model test for the genotype data for the studies yielded a statistically significant result $\left(\mathrm{Q}=13.91\right.$, $\mathrm{p}$ value $<0.001, \mathrm{I}^{2}=49.70$, tau squared $\left.=0.053\right)$ 
act by affecting the expression of TCF7L2. T allele of variant rs7903146 of TCF7L2 gene is less prone to be bound by protein factors like GATA3 in hepatic cell lines and hence there is aberrant TCF7L2 expression in patients with TT genotype [10]. Similar effect was seen in other cell lines like HepG2 (liver), 3T3-L1 (adipocytes), and $\beta$-TC3 ( $\beta$ cells) where rs7903146 T allele bound with lesser efficiency to protein factors compared to alternative $\mathrm{C}$ allele. Our group has recently shown the genetic association of transcription factor 7 like 2 (TCF7L2) gene, a part of $\beta$-catenin signaling cascade, to be associated with impairment of wound healing in T2DM individuals [11]. In the present study, a Meta analysis was done to systematically review the association of this SNP with common secondary complication associated with T2DM by combining our data with data from previous studies.

\section{MATERIALS AND METHODS}

\subsection{Literature search}

Different databases like PubMed, OMIM, ISI web of science and Embase were comprehensively searched for the association of SNP rs 7903146 polymorphism with secondary complication of T2DM published after January 2009 till August 2014. The search strategy was based on combination of keywords like "transcription factor 7 - like 2" or "TCF7L2" or "rs 7903146" polymorphism with "secondary complication of diabetes" or "retinopathy" or "neuropathy" or "cardiomyopathy" or "wound healing impairment". All original articles which reported data from original studies were included in this Meta analysis.

\subsection{Inclusion and exclusion criteria}

Thorough reviewing of full text of each short listed article was done to fetch necessary information for Meta analysis. The inclusion criteria of the study eligible for Meta analysis was chosen so that trails being (i) independent case control study, (ii) with sufficient information required for calculation of Odds ratio, (iii) good resolution SNP genotyped (iv) similar in statistical and purpose methodology were included for Meta analysis. The exclusion criteria of the study was (i) T2DM occurrence after any transplantation event, (ii) any metabolic associated circumstance like obesity or inflammation, (iii) evidence of auto immunity like that in type 1 diabetes or LADA (latent autoimmune diabetes in adults), (iv) involvement of one or other Genetic disorder.

\subsection{Raw Data Extraction}

Genotype data for SNP rs 7903146 polymorphism were extracted according to standard extraction form (Fig. 1). Data for secondary complications of T2DM were collected. Tabulation of the arranged data was then done with particular details regarding the first author, year of publication, sample size, ethnicity of the study population, and genotype frequency.

\subsection{Quantitative data synthesis}

For Meta analysis all statistical test were performed using software comprehensive Meta Analysis V2. Odds ratio (ORs) was chosen as effect size to find secondary complications of T2DM associated with mutant genotype (TT) of SNP rs 7903146 polymorphism.

\section{Funnel Plot of Standard Error by Log odds ratio}

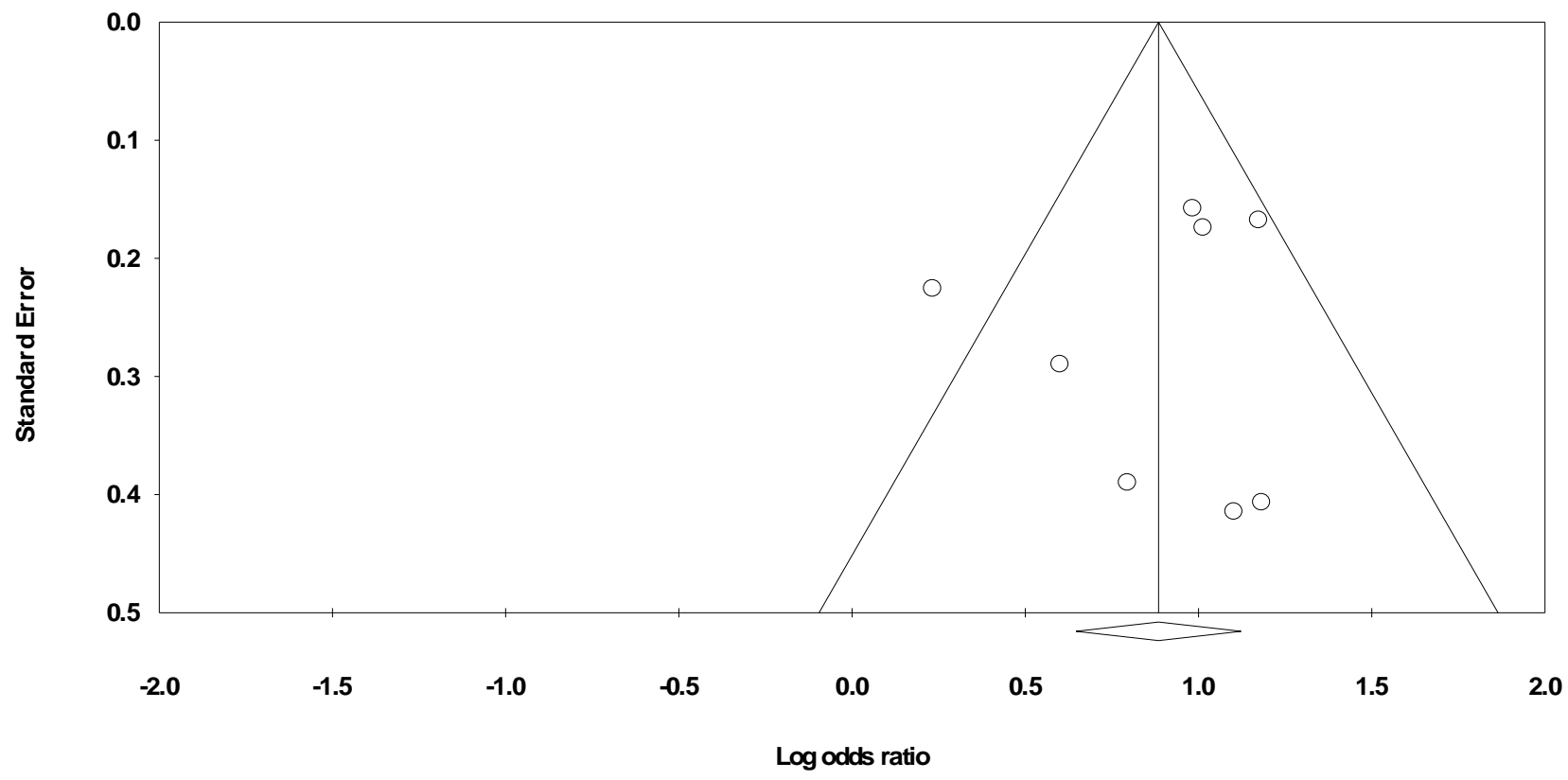

Fig. 3: Publication bias of studies was calculated using funnel plot of standard error calculated by using log odds ratio. Funnel plot of precision based on log odds ratio was created using various statistical tools such as Egger's regression intercept, Duvall and Tweedie trim and fill procedure Classic fail safe ' $\mathrm{N}$ ' to nullify the effect of publication bias owing to small studies. The possibility of publication bias was rejected due to the evidence of symmetrical distribution of studies on funnel plot. 
Cochran's $\chi^{2}$ test (Q test) was used to evaluate heterogeneity between studies; a threshold $\mathrm{P}$ value of 0.1 was considered statistically significant, and the inconsistency value $\left(\mathrm{I}^{2}\right)$ test was also conducted to evaluate heterogeneity of studies. Since no evidence of heterogeneity was found in the studies, fixed model was utilized to calculate the $\mathrm{p}$ value and effect size of the study. High resolution plots were generated equivalent to ORs and $\mathrm{CI}$ at $95 \%$ for fixed as well as random effects models. Sensitivity analysis was performed to check the validation and robustness of method utilized in the analysis. Funnel plot of precision based on log odds ratio was created using various statistical tools such as Egger's regression intercept, Duvall and Tweedie trim and fill procedure Classic fail safe ' $\mathrm{N}$ ' to nullify the effect of publication bias owing to small studies. Classic Fail safe ' $N$ ' suggests the number of null studies which have to be included to subsidize the effect to non-significant. Egger's test for intercept provides the overall estimate of the effect size.

\section{RESULTS}

A total of 5 research articles comprised of 8 studies including our previous study were found to be eligible on our search criteria. Buraczynska et al. [12] reported association of rs 7903146 with nephropathy, diabetic retinopathy and Cardiomyopathy, while Ciccacci et al. [13] associated rs 7903146 with diabetic retinopathy and CAD. Hence there were total 8 eligible studies including our study comprised of a total of 2060 cases with secondary complication of T2DM and 3049 age matched controls without T2DM. The genotypes were classified into high risk (TT) and low risk $(\mathrm{CC}+\mathrm{CT})$ for statistical calculations. The ' $\mathrm{I}^{2}$ ' value of $49.70 \%$ for between studies comparison showed low level of true heterogeneity. The fixed model test for the genotype data for the studies yielded a statistically significant result $\left(\mathrm{Q}=13.91, \mathrm{p}\right.$ value $<0.001, \mathrm{I}^{2}=$ 49.70, tau squared $=0.053$ ).

Egger's test for intercept showed no evidence of publication bias (intercept $=-0.76, \mathrm{SE}=1.50,95 \% \mathrm{CI}=-4.44$ to 2.90 , p 2 tailed $=0.62$ ). Classic Fail safe ' $N$ ' $=223$, missing from the analysis for every published study included, which further supports absence of publication bias. Funnel plot of standard error by $\log$ odds ratio was also generated (Fig. 2). The possibility of publication bias was rejected due to the evidence of symmetrical distribution of studies on funnel plot (Fig. 3).

\section{CONCLUSION}

Wnt signaling works via disheveled receptors and transcription factors like $\beta$-catenin and TCF7L2 to modulate the expression of downstream target genes like IL1, TNF- $\alpha$, FGF, KGF and VEGF [14]. The transcription factor 7 like 2 gene product, a High mobility group box transcription factor, is a part of $\beta$-catenin signaling cascade [15]. Duggirala et al. [16] described about a region on chromosome $10 \mathrm{q}$ that was linked to T2DM. Further Grant et al. [17] showed a strong association between a microsatellite marker located within intron 3 of TCF7L2 and following that study an another study revealed that SNP rs 7903146 allele $\mathrm{T}$ within the gene TCF7L2 is in linkage disequilibrium with this microsatellite marker [18]. The association of this variant with T2DM has already been established in different cohorts of world including Asian populations like India and Pakistan [19-22]. This TCF7L2 gene polymorphism has been already shown to be involved in secondary complications of diabetes like nephropathy, neuropathy, retinopathy and cardiovascular disease $[12,13,23]$. TCF7L2 may affect $\beta$ - cell function either by modulating $\beta$-cell response to glucose or by modulating the incretin action or secretion [24]. The minor T allele of this SNP is strongly associated with HOMA-B level and hence results in diabetic condition either by affecting function of pancreatic $\beta$-cell or by impaired GLP-1 induced insulin secretion $[25,26]$. Functional studies show that T allele of SNP rs 7903146 is less prone to be bound by protein factors like GATA3 in hepatic cell lines and hence there is aberrant TCF7L2 expression in patients with TT genotype [27]. Cauchi et al. showed that this polymorphism have no impact on expression of specific TCF7L2 isoforms in Non Glucose Tolerant (NGT) subjects [28]. Further he added that in T allele NGT carriers of this SNP has low TCF7L2 expression in their adipose tissue and anergetic nutrition i.e. high calorie diet may help in increasing TCF7L2 expression. Interestingly the minor T allele of SNP is neither associated with higher BMI nor it has been associated with risk lipid profile i.e. the patients with this $\mathrm{T}$ allele has lesser chance to develop metabolic syndrome [29]. To the best of our knowledge only 5 studies including our study have evaluated the association of SNP rs 7903146 of TCF7L2 gene with secondary complications in diabetes. Sousa et al. in 2009 [30] reported the association of TCF7L2 polymorphism rs7903146 and coronary artery disease in diabetic and non-diabetic subjects in two independent population of Brazil, but the association was non significant in case of diabetic patients. In another study Buraczynska et al. in 2011 [12] showed the association of this variant with diabetic nephropathy, diabetic retinopathy and cardiovascular disease among Caucasians of Polish origin. They also concluded that $\mathrm{T}$ allele of this polymorphism was strongly associated with nephropathy, particularly in early onset of diabetes. Muendlein et al. in 2011 [22] investigated the association between this polymorphism and angiographically determined CAD in diabetic and non-diabetic patients in whites of Austria. They also found that this variant was significantly associated with angiographically diagnosed CAD, specifically in patients with T2DM. In a study conducted by Ciccacci et al. in 2012 [13], the authors studied this gene polymorphism in the pathogenesis of diabetic retinopathy and neuropathy. They concluded that a strong correlation was present between the rs7903146 variant of TCF7L2 and the presence of cardiovascular autonomic neuropathy and diabetic retinopathy. Our group has analyzed the association of TT genotype of SNP rs 7903146 with another secondary complication of T2DM which is impairment in healing of wounds in T2DM patients and found a significant association of TT genotype with wound healing 
impairment [11]. Based on above previous studies and combining our own study we conducted a Meta analysis for analyzing the association of this polymorphism with secondary complication of T2DM. Meta analysis of all five studies also showed highly significant association of risk genotype TT with development of secondary complication in T2DM.

\section{CONFLICT OF INTEREST}

The authors declare no conflict of interest.

\section{ACKNOWLEDGEMENT}

This work was funded by Department of science and technology, New Delhi, India. Financial assistance by Department of Biotechnology, Ministry of Science and Technology, New Delhi, India in form of Senior Research fellowship to the first author is thankfully acknowledged.

\section{REFERENCES}

1. Singh K, Agrawal NK, Gupta SK, Singh K. A functional SNP$1562 \mathrm{C}>\mathrm{T}$ in the matrix metalloproteinases -9 promoter is associated with type 2 diabetes and diabetic foot ulcers. Int $\mathrm{J}$ Lower Extrem Wounds. 2013; 12(3): 199-204

2. World Health Organization. Definition, Diagnosis and Classification of Diabetes Mellitus and Its Complications: Report of a WHO Consultation (WHO/NCD/NCS/99.2). Geneva, Switzerland: World Health Organization; 1999.

3. Ali O. Genetics of type 2 diabetes. World J Diabetes. 2013; 4(4): 114-123.

4. Chauhan G, Spurgeon CJ, Tabassum R et al. Impact of Common Variants of PPARG, KCNJ11, TCF7L2, SLC30A8, HHEX, CDKN2A, IGF2BP2, and CDKAL1 on the Risk of Type 2 Diabetes in 5,164 Indians. Diabetes. 2010; 59(8): 2068-2074

5. Singh K, Agrawal NK, Gupta SK, Mohan G, Chaturvedi S and Singh K. Differential expression of Matrix metalloproteinase- 9 gene in wounds of T2DM cases with susceptible $-1562 \mathrm{C}>\mathrm{T}$ genotypes and wound severity. Int J Lower Extrem Wounds. 2014; 13(2): 94-102.

6. Laeremans H, Rensen SS, Harry CJ et al.: Wnt/frizzled signalling modulates the migration and differentiation of immortalized cardiac fibroblasts. Cardiovascular Research. 2010; 87: 514-523.

7. Cheon SS, Wei Q, Gurung A et al.: Beta-catenin regulates wound size and mediates the effect of TGF-beta in cutaneous healing. FASEB Journal. 2006; 20: 692-701.

8. Prunier C, Hocevar BA, Howe PH et al.: Wnt signaling: physiology and pathology. Growth Factors. 2004; 22(3):141-150.

9. Hansson $\mathrm{O}$, Zhou $\mathrm{Y}$, Renstrom $\mathrm{E}$ et al.: Molecular function of TCF7L2: Consequences of TCF7L2 splicing for molecular function and risk for type 2 diabetes. Curr Diab Rep. 2010; 10(6): 444-451.

10. Lyssenko V, Lupi R, Marchetti $\mathrm{P}$ et al.: Mechanisms by which common variants in the TCF7L2 gene increase risk of type 2 diabetes. J. Clin. Invest. 2007; 117: 2155-2163.

11. Singh K, Agrawal NK, Gupta SK, Singh K. Association of Variant rs7903146 (C/T) Single nucleotide polymorphism of TCF7L2 gene with impairment in wound healing among north Indian Type 2 Diabetes population: A Case-Control Study. Int J Lower Extrem Wounds. 2013; 12(4): 310-315.

12. Buraczynska M, Swatowski A, Markowska-Gosik $\mathrm{D}$ et al.: Transcription factor 7-like 2 (TCF7L2) gene polymorphism and complication/comorbidity profile in type 2 diabetes patients. Diabetes research and clinical practice. 2011; 93: 390-395.

13. Ciccacci C, Fusco DD, Cacciotti L et al.: TCF7L2 gene polymorphisms and type 2 diabetes: association with diabetic retinopathy and cardiovascular autonomic neuropathy. Acta Diabetol. 2012; DOI 10.1007/s00592-012-0418-x.

14. Zhang DL, Gu LJ, Liu L et al. Effect of Wnt signaling pathway on wound healing. Biochemical and Biophysical Research Communications. 2009; 378: 149-151.

15. Yi F, Brubaker PL, Jin T et al. TCF-4 mediates cell type-specific regulation of proglucagon gene expression by beta-catenin and glycogen synthase kinase-3 beta. J Biol Chem. 2005; 280(2): 14571464.

16. Duggirala R, Blangero J, Almasy L: Linkage of Type 2 Diabetes Mellitus and of Age at Onset to a Genetic Location on Chromosome 10q in Mexican Americans. Am. J. Hum. Genet. 1999; 64: 11271140

17. Grant SF, Thorleifsson G, Reynisdottir I et al. Variant of transcription factor 7-like 2 TCF7L2 gene confers risk of type 2 diabetes. Nature Genetics. 2006; 38: 320-323.

18. Cauchi S, El Achhab Y, Choquet $\mathrm{H}$ et al.: TCF7L2 is reproducibly associated with type 2 diabetes in various ethnic groups: A global meta-analysis. J Mol Med. 2007; 85: 777-782.

19. Bodhini D, Radha V, Dhar M et al.: The $\operatorname{rs} 12255372(\mathrm{G} / \mathrm{T})$ and rs 7903146(C/T) polymorphisms of the TCF7L2 gene are associated with type 2 diabetes mellitus in Asian Indians. Metabolism. 2007; 56: 1174-1178.

20. Chandak GR, Janipalli CS, Bhaskar S et al. Common variants in the TCF7L2 gene are strongly associated with type 2 diabetes mellitus in the Indian population. Diabetologia. 2007; 50: 63-67.

21. Rees SD, Bellary S, Britten AC et al. Common variants of the TCF7L2 gene are associated with increased risk of type 2 diabetes mellitus in a UK-resident South Asian population. BMC Med Genet. 2008; 9:8.

22. Muendlein A, Saely CH, Geller-Rhomberg S et al. Single Nucleotide Polymorphisms of TCF7L2 Are Linked to Diabetic Coronary Atherosclerosis. PLoS ONE. 2011; 6(3): e17978.

23. Jin T and Liu L: The Wnt Signaling Pathway Effector TCF7L2 and Type 2 Diabetes Mellitus. Mol Endocrinol. 2008; 22(11): 23832392.

24. Saxena R, Gianniny L, Burtt NP et al. Common single nucleotide polymorphisms in TCF7L2 are reproducibly associated with type 2 diabetes and reduce the insulin response to glucose in nondiabetic individuals. Diabetes. 2006; 55: 2890-2895.

25. Yi F, Brubaker PL, Jin T et al. TCF-4 mediates cell type-specific regulation of proglucagon gene expression by beta-catenin and glycogen synthase kinase-3beta. J Biol Chem. 2005; 280: 14571464.

26. Cauchi S, Choquet H, Gutiérrez-Aguilar R et al:: Effects of TCF7L2 polymorphisms on obesity in European populations. Obesity 2008, 16(2):476-482

27. Cauchi S and Froguel P. TCF7L2 Genetic Defect and Type 2 Diabetes. Current Diabetes Reports. 2008; 8:149-155.

28. Melzer D, Murray A, Hurst AJ et al. Effects of the diabetes linked TCF7L2 polymorphism in a representative older population. BMC Med. 2006; 4:34.

29. Sousa AGP, Marquezine GF, Lemos PA et al. TCF7L2 Polymorphism rs7903146 Is Associated with Coronary Artery Disease Severity and Mortality. PLoS ONE. 2009; 4(11): e7697.

\section{How to cite this article:}

Kanhaiya Singh, NK Agrawal, SK Gupta, Kiran Singh. Meta analysis of variant rs 7903146 of TCF7L2 with secondary complications of Type 2 Diabetes Mellitus. J App Biol Biotech. 2014; 2 (05): 001-005. 